\title{
Information aus der Fachkommission für Dopingbekämpfung (FDB)
}

\author{
M. Kamber ${ }^{a}$, N. Mahler $^{a}$, M. Strupler ${ }^{b}$
}

\section{Neue Dopingliste ab 1. Januar 2005}

Eine neue Dopingliste tritt auf den 1. Januar 2005 in Kraft. Es ist bereits das zweite Mal, dass die Welt-Anti-Doping-Agentur (WADA) alleine für deren Inhalt verantwortlich zeichnet. Gemäss dem WADA-Code und dem Internationalen Standard zur Erstellung einer Dopingliste sollen nur Wirkstoffe und Methoden in der Liste erscheinen, die mindestens zwei von drei Kriterien entsprechen:

- gemäss medizinischen oder anderen wissenschaftlichen Erkenntnissen hat der Wirkstoff oder die Methode das Potential zur Leistungssteigerung im Sport;

- gemäss medizinischen oder anderen wissenschaftlichen Erkenntnissen besitzt der Wirkstoff oder die Methode ein aktuelles oder potenzielles Gesundheitsrisiko;

- gemäss WADA verstösst die Anwendung des Wirkstoffs oder der Methode gegen die Ethik im Sport (spirit of sport).

In einem breiten Vernehmlassungsverfahren konnten interessierte Partnerorganisationen im Sommer 2004 zum ersten Entwurf der Dopingliste 2005 Stellung nehmen. Die Fachkommission für Dopingbekämpfung (FDB) hatte diese Gelegenheit benutzt und verschiedene Anregungen eingereicht. So hat sie Klärungen im Zusammenhang mit bestimmten Formulierungen, z. B. zu Adrenalin, zum Verbot intravenöser Infusionen und zu Auflistungen bestimmter Wirkstoffe verlangt. Insbesondere wollte die FDB Glukokortikoide nur in bestimmten Sportarten verbieten (Verschiebung in die Klasse der «in gewissen Sportarten verbotenen Wirkstoffe») und die Cannabinoide ganz von der Liste streichen (oder allenfalls nur ein Verbot bei bestimmten Sportarten). Einige der Bemerkungen wurden scheinbar aufgenommen. Leider hat die WADA weder Rückmeldung über die erhaltenen Vorschläge gemacht noch Argumente oder Erklärungen zu ihren Entscheidungen geliefert.

Korrespondenz:

Dr. med. Matthias Strupler

Schweizer Paraplegiker-Forschung AG Institut für Sportmedizin CH-6207 Nottwil

\section{Neue Einteilung der Dopingliste}

Die Dopingliste wurde wiederum neu eingeteilt. Ab 1. Januar 2005 werden zuerst die Wirkstoffe und Methoden genannt, die jederzeit, im Wettkampf wie ausserhalb der Wettkämpfe, verboten sind. Im Vergleich zur Liste 2004 sind die verbotenen Wirkstoffgruppen neu geordnet und in einer anderen Reihenfolge dargestellt. Die Anzahl der Wirkstoffgruppen und die Anzahl der verbotenen Methoden haben sich aber nicht geändert.

Wie in der Liste 2004 werden nur noch gewisse Grenzwerte (z.B. für Ephedrin, Cathin, Salbutamol) in der Liste 2005 genannt werden. Andere Grenzwerte (wie z.B. für Cannabinoide, Morphin, Nandrolon) finden sich hingegen nur noch in technischen Dokumenten für die Labors. Dies soll der Auffassung vorbeugen, dass die Anwendung dieser Wirkstoffe bis zu ihrem Grenzwerten erlaubt sei. Im Sinne der möglichst grossen Transparenz ist dies aber keine optimale Lösung.

Bei einigen Wirkstoffklassen, die keine abschliessende Aufzählung der Beispiele enthalten, wird der bisherige Begriff «pharmakologisch» durch «biologisch» ersetzt.

\section{Änderungen bei den verbotene Wirkstoffen}

\section{Anabolika}

Die folgenden neun anabolandrogenen Steroide (ASS) werden namentlich in die Gruppe der exogenen AAS aufgenommen: 18 $\alpha$-Homo-17 $\beta$ hydroxyestr-4-en-3-on, Calusteron, Ethylestrenol, Furazabol, Methyldienolon, Methyltrienolon, Norclostebol, Stenbolon und Tetrahydrogestrinon. Zusätzlich werden in einer anderen Aufzählung zahlreiche Metaboliten und Isomere endogener (körpereigener) Steroide zur Liste hinzugefügt.

Die endogenen AAS sind neu in einer abgeschlossenen Liste aufgeführt.

Unverständlicherweise (und im Entwurf vom Juli 2004, der uns zur Stellungnahme vorlag, noch nicht so beschrieben) wird nun vorgeschrieben, dass bei einem Verhältnis von Testosteron zu Epitestosteron von $4 \mathrm{zu} 1$ (bisher 6 $\mathrm{zu}$ 1) Zusatzuntersuchungen durchgeführt werden müssen. Dies wird den administrativen und finanziellen Aufwand in diesem Bereich für die FDB erheblich erhöhen. 
Unter «andere anabole Wirkstoffe» wird neu Zilpaterol verboten.

Hormone und andere verwandte Substanzen Diese Wirkstoffgruppe wurde umbenannt. Sie hiess 2004 noch «Peptidhormone». Die wichtigste Änderung ist hier, dass Wirkstoffe wie HCG und Gonadotropine neu für alle und nicht wie bisher nur bei Männern verboten sind. Wie und wem ein «positives» Laborresultat für HCG bei einer Athletin mitgeteilt wird, muss noch genau festgelegt werden. Eine mögliche Schwangerschaft gehört unseres Erachtens zu den besonders schützenswerten persönlichen Daten.

Neu in der Liste sind auch die Mechanowachstumsfaktoren (MGFs) und die Zusammenfassung der bisherigen Subkategorien der Gonadotropine zu einer einzigen.

\section{Beta-2-Agonisten}

Neu ist, dass Beta-2-Agonisten nun jederzeit verboten sind, d.h. innner- und ausserhalb von Wettkämpfen. Wie bisher werden lediglich vier Wirkstoffe, nämlich Formoterol, Salbutamol, Salmeterol und Terbutalin, mit einer Ausnahmebewilligung zu therapeutischen Zwecken (ATZ, vereinfachtes Verfahren) zur Inhalation erlaubt. Administrativ wird diese Änderung keinen Mehraufwand bringen, waren doch bereits bisher die meisten ATZ für Beta-2-Agonisten nicht nur für die Wettkampfphase beantragt worden.

\section{Antiöstrogene Wirkstoffe}

Diese Klasse wird neu in drei Subgruppen unterteilt. Die Wirkstoffe Anastrozol, Letrozol, Aminogluthemid, Exemestan, Formestan, Testolakton, Raloxifen, Toremifen und Fulvestrant werden neu als Beispiele namentlich aufgeführt. Die bisher nur für Männer verbotenen Wirkstoffe sind neu auch für Frauen verboten.

\section{Diuretika und andere maskierende Wirkstoffe}

Der Titel der Gruppe wurde geändert. Bei den Diuretika werden namentlich Metolazon hinzugefügt und Mersalyl entfernt. Als neu verbotene maskierende Wirkstoffgruppe werden die Inhibitoren der Alpha-Reduktase (z.B. Finasterid und Dutasterid) aufgeführt.

\section{Stimulanzien}

Neu auf der Liste sind namentlich Famprofazon und Fencamin. Ein Zusatz erklärt den Status von Adrenalin: Adrenalin, angewendet zusammen mit Lokalanästhetika oder in lokalen Präparaten (z.B. für Nase oder Auge), ist nicht verboten. Zudem wird Bupropion ins Überwachungsprogramm 2005 aufgenommen.

\section{Narkotika}

Fentanyl und seine Derivate wurde in die abgeschlossene Aufzählung der Wirkstoffe aufgenommen.

\section{Cannabinoide}

Bei den Cannabinoiden hat sich gegenüber der Liste 2004 nichts geändert. Sie bleiben wie bisher im Wettkampf verboten. Im Jahr 2004 hatten wir in der Schweiz mehrere Dopingfälle mit Cannabis. Teilweise wurde argumentiert, dass die positiven Resultate lediglich durch Passivrauchen zustande kamen. Eine Athletin hat gegen die erstinstanzliche Verurteilung mit dieser Begründung ans Tribunal arbitral du sport (TAS) appelliert und verloren. Die FDB empfiehlt deshalb den Athletinnen und Athleten, während der Wettkampfphase vollständig auf den Konsum von Cannabinoiden (Cannabis, Haschisch, Marihuana usw.) zu verzichten. Vorsicht ist auch bei starker Gefährdung durch Passivrauchen geboten (kleine Räume mit viel Haschischrauch, schlechter Lüftung, lange Aufenthaltszeit in diesen Räumen).

\section{Glukokortikoide}

Das Jahr 2004 war geprägt von grossem administrativem Aufwand wegen der vielen Anträge auf Ausnahmebewilligungen zu therapeutischen Zwecken (ATZ), die Glukokortikoide betrafen. Im Jahr 2005 wird sich die Situation insofern etwas entschärfen, als die dermatologische Anwendung von Glukokortikoiden wieder erlaubt sein wird und deshalb keiner ATZ mehr bedarf. Die lokale, topische und intraartikuläre Anwendung benötigt für Wettkämpfe eine ATZ nach vereinfachtem Verfahren. Die systemische Anwendung (per oral oder intramuskulär) ist weiterhin verboten. Das bedeutet, dass eine entsprechende Therapie nur mit einer Bewilligung der FDB nach einem Antrag gemäss dem Standardverfahren möglich ist.

\section{Änderungen bei den verbotene Methoden}

\section{Chemische und physikalische Manipulation}

Der früher verwendete Ausdruck «pharmakologisch» wurde aus dem Titel gestrichen, da sich die entsprechenden Substanzen nun in der Klasse der Diuretika und anderen maskierenden Wirkstoffe befinden.

Neu wurden von der WADA intravenöse Infusionen als Dopingmethode verboten, wenn sie nicht zur akuten medizinischen Behandlung dienen. Nachfragen bei der WADA haben ergeben, dass hiermit nicht eine Bevormundung von Ärztinnen und Ärzten beabsichtigt ist und das 
Verbot vor allem Infusionen zur Blutverdünnung betrifft. Zudem habe das Verbot auch eine erzieherische Wirkung: sie soll für das Problem der nicht medizinisch indizierten Infusionen im Sport sensibilisieren. Es soll auch einen Schutz für Athletinnen und Athleten darstellen, da mittels Infusionen leichter unbemerkt Dopingmittel verabreicht werden können. Repräsentanten der WADA haben versprochen, entsprechende Präzisierungen zu liefern.

\section{Registrierter Kontrollpool}

Gemäss WADA-Code und dem entsprechend angepassten Dopingstatut von Swiss Olympic werden nationale und internationale Kontrollpools gebildet. Generell gelten die Dopingbestimmungen für alle Sporttreibenden. Lediglich die administrativen Anforderungen sind anders, je nachdem, ob man einem Kontrollpool angehört oder nicht. Alle Sporttreibenden, die einem nationalen oder internationalen Kontrollpool angehören (dies betrifft nationale/internationale Spitzensportlerinnen und -sportler), werden von ihren Sportverbänden über ihre Zugehörigkeit zum Pool informiert. Für sie gilt die
Meldepflicht ihres Aufenthaltsortes, Bedingungen beim Rücktritt (Entscheid, ob sie im Kontrollpool bleiben wollen oder nicht) und die Regelungen bezüglich ATZ (ATZ müssen vorgängig eingereicht werden). Alle anderen Sporttreibenden können eine ATZ freiwillig einreichen. Bei Vorliegen eines positiven Analysenresultates oder bei Bedarf kann die FDB die ATZ zudem nachträglich anfordern.

\section{Neu gestaltete Website}

Die Website www.dopinginfo.ch erfreut sich grosser Beliebtheit. Die Benutzerzahlen steigen stetig. Damit sie noch besser den verschiedenen Bedürfnisgruppen entspricht, wurde sie kürzlich angepasst und soll Anfang 2005 sukzessive in neuer Form zur Verfügung stehen. Die FDB hat zudem beschlossen, dass sie die offizielle Website der Dopingbekämpfung Schweiz sein wird. Die aktuelle Dopingliste und die Formulare für die Ausnahmebewilligung zu therapeutischen Zwekken können Sie von dieser Website herunterladen. Dies ist der Grund, weshalb die Dopingliste nicht mehr der Ärztezeitung beigelegt wird. 